\section{A program for analyzing temporal pattern intervals in repetitive, animal sounds}

\author{
JOHN A. DOHERTY \\ Villanova University, Villanova, Pennsylvania
}

Software for measuring temporal pattern intervals of repetitive animal sounds is described. The program measures time intervals of digitized sounds, plots frequency distributions of these intervals, and calculates descriptive statistics. The program is written in THINK Pascal and is currently interfaced with a sound digitization and analysis software package for the Macintosh computer (SoundWave, Impulse, Inc.). However, the algorithms can be incorporated in any hardware/software environment for the digitization and analysis of a variety of repetitive, pulsatile animal sounds.

Studies of animal communication are often concerned with the temporal analysis of repetitive acoustic signals. Such signals can be found throughout the animal kingdom, in, for example, acoustic insects, frogs, birds, and mammals. The analysis of these repetitive sounds usually includes measurements of sound-pulse durations and repetition rates.

In this paper, I describe algorithms for the rapid temporal analysis of repetitive animal sounds. The program was written in THINK Pascal on the Apple Macintosh computer and was combined with an inexpensive, commercially available sound analysis software package for the Macintosh (SoundWave, Impulse, Inc.). The software was originally developed for the analysis of tape recordings of the calling songs of crickets. The program described in this paper measures the time intervals of digitized sounds, plots histograms of these intervals, and reports descriptive statistics. Although the software was developed primarily for measuring repetitive animal sounds on the Macintosh in conjunction with Impulse's SoundWave software, the developed algorithms could be modified to analyze any digitized sound files generated by several inexpensive, commercially available hardware/software packages for sound digitization and analysis.

\section{Cricket Stridulation and Some Special \\ Problems in Sound Analysis}

Male crickets produce a rhythmic series of pulses by the process of forewing stridulation (Huber \& Thorson, 1985). In stridulation, the male moves the hardened edge on one of its forewings (called the scraper) over a row of teeth (called the file) on the other forewing. Each time the scraper is moved over the entire row of teeth on the file, a sound pulse is produced-a process analogous to

Correspondence should be addressed to John A. Doherty, Department of Biology, Villanova University, Villanova, PA 19085. BitNet: DOHERTY@VUVAXCOM. moving one's fingernail over the teeth of a comb. The frequency or pitch of each sound pulse approximates a pure sinusoid, with pulse carrier frequencies ranging from 3 to $8 \mathrm{kHz}$, depending on the species. Some species of crickets produce a continuous train of pulses (called trills), whereas other species package the sound pulses into rhythmic pulse trains called chirps (see Figure 1A). The temporal patterning of sound pulses in male calling songs is species-specific and is important for attracting conspecific females for mating (positive phonotaxis; see Doherty \& Hoy, 1985; Huber \& Thorson, 1985).

Studies of cricket sound communication often entail the measurement of pulse durations and pulse repetition rates of male calling songs. The software described in this paper measures these intervals from amplitude envelopes of previously digitized pulses that have been filtered by downsampling and rectified by eliminating the belowbaseline, negative deflections of the pulse amplitude envelope. These interval measurements are made at a userselectable trigger amplitude. A major problem that had to be overcome in the development of these interval measurement algorithms was the presence of false pulse rise and fall times that are produced either by tape drop-outs or, in the case of wing stridulation by crickets, by the scraper's skipping over several teeth of the file (for examples of this, see Pulses 1 and 2 in Figure 1). This yielded shortened false pulses, which had to be detected and avoided by the interval measurement algorithm.

\section{Sound Digitization}

Several inexpensive sound digitizers can be purchased for digitizing animal sounds in conjunction with the Macintosh computer. The two digitizers that I use routinely and interchangeably in my Macintosh sound analysis system are Impulse's SoundWave digitizer and Farallon's MacRecorder. Both digitizers can sample sounds at several rates between 5 and $22 \mathrm{kHz}$. Since cricket calling songs approximate a pure sinusoid in spectral quality and dominant frequencies of most of these songs range between 3 and $5 \mathrm{kHz}$, I sample the sound at $22 \mathrm{kHz}$ for spectral analysis. For temporal analysis (i.e., pulse duration, rate, etc.), I sample sounds at $5.5 \mathrm{kHz}$. This low sampling rate is adequate for resolving pulse amplitude envelopes in cricket calling songs (cricket sound pulses usually have durations ranging between 10 and $30 \mathrm{msec}$ and repetition intervals of $20-60 \mathrm{msec}$ ).

The sound analysis program described below analyzes sounds that have been digitized at $5.5 \mathrm{kHz}$. As a matter of convenience, my program is embedded in Impulse's SoundWave software, which provides procedures for interfacing SoundWave with customized code. The program is not limited to the SoundWave software environment, however. Sounds can be digitized by any digitizer and the sound analysis program can be modified to read in any previously digitized sound that has been saved as an 
A)

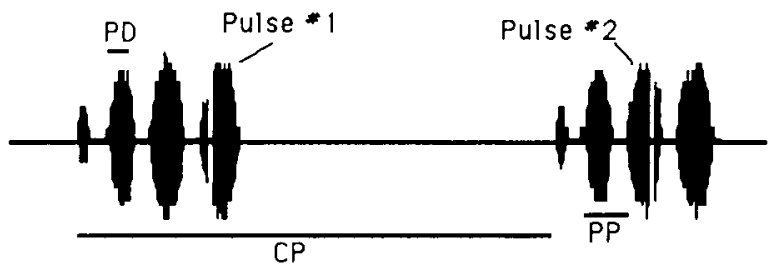

B)

- Pulse Beginning

O Pulse End

Chirp Beginning

$\uparrow$ Criterion Silent Interval

(a TRUE Baseline condition)
C)

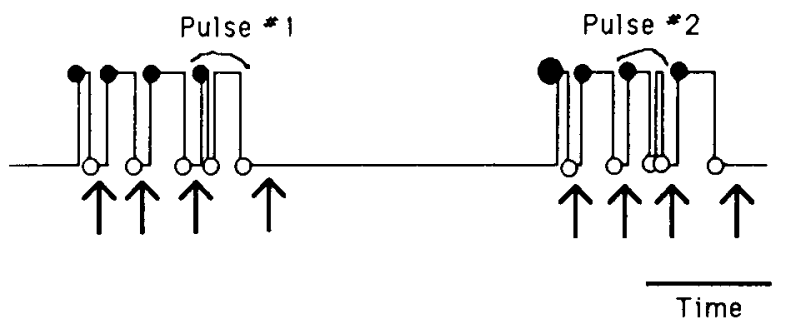

Figure 1. Oscillograms that show various transformations of digitized sound, (A) Oscillogram of a previously digitized calling song produced by a male field cricket of the species Gryllus veletis. The oscillogram shows two calling song chirps, each composed of four sound pulses. The numbered pulses (1 and 2) show false pulse conditions due to tape drop-out or the male's forewing scraper skipping over portions of the file located on the other forewing. PD = Pulse Duration, PP = Pulse Period, CP = Chirp Period. (B) The carrier frequency of the sound in $(A)$ is filtered by downsampling and the pulse is rectified by eliminating the portion of pulse below baseline. The horizontal line just above the baseline shows the user-adjustable trigger level. (C) The rectified signal (B) is transformed into a square pulse. The presence of a pulse rise or fall above or below the trigger level (B) is indicated by solid and open circles. Pulse intervals are only measured and stored when a silent interval greater than a minimum criterion duration is encountered (silent intervals that meet this criterion are shown by the vertical arrows). Notice that Pulse 1 has a false rise near the beginning of the pulse. Pulse 2 has a false fall near the end of the pulse.

ASCII text file (see descriptions of the SampleSoundFile and ReadSoundFile procedures).

\section{General Description of the \\ Sound Analysis Program}

The program was written in THINK Pascal on the Macintosh computer. Important code segments are found in the Appendix. The program takes any previously digitized sound (as in Figures 1A and 2) and transforms this sound as follows:

1. The carrier frequency of the sound pulse is rectified and filtered, so that all that remains is the amplitude envelope of the pulse above baseline (see Figures 1A, 1B, and 2).

2. The user is allowed to select the measurement trigger level, so that it is just above the baseline noise (Figure 1B).

3. The selected trigger level is used to transform the amplitude envelope into simple square pulses (Figure 1C).

4. The time intervals of the square pulses are measured (pulse duration, pulse period, chirp period; see
Figure 1C). False pulse rise and fall times (inverse of pulse repetition rate) are detected and their measurement avoided.

5. The intervals in frequency histograms are plotted, and descriptive statistics are reported (Figure 3).

The program is a THINK Pascal unit called SoundAnalysis, which is linked to a pull-down menu in the SoundWave program. Detailed information about the linkage of customized code to the SoundWave program is found in the accompanying instruction manual. The SoundAnalysis unit has several variable types for interfacing with the SoundWave program and just one procedure called Main. Within the Main procedure are several subprocedures that do the transformations of the digitized sound required for interval measurements and analysis. The names of the principal subprocedures and their primary functions are:

Initialize;

$\{$ Gets sound pointers and resources. Initializes other parameters. 


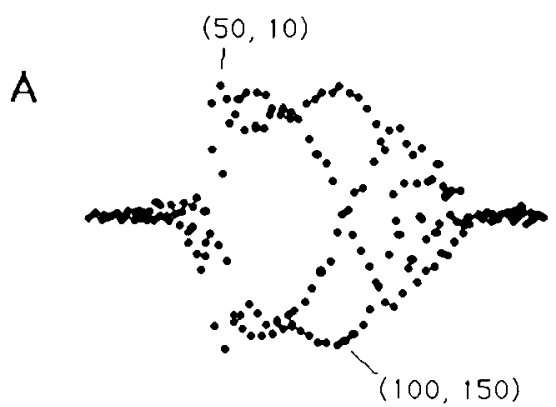

B

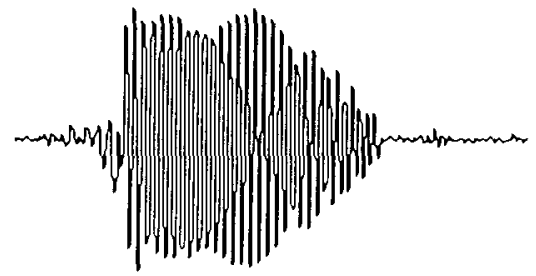

C

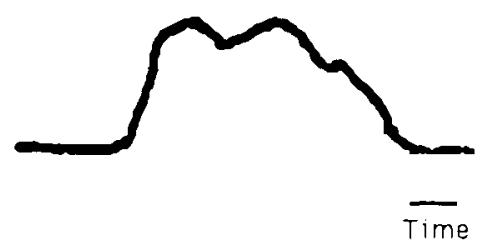

Figure 2. Oscillograms showing the transformation from a digitized sound pulse $(A)$ to a pulse that has been filtered and rectified (C). The pulse in the middle oscillogram (B) is the same as that in the upper oscillogram (A), except that lines have been drawn between consecutive sample points. The pulse in (B) is filtered by downsampling by writing only every sixth sample point in the transformation. It is rectified by eliminating pulse excursions below baseline (see the description of the ReadSoundFile procedure). On the computer monitor, the abscissa of sample point coordinates in (B) increases, going from left to right; the ordinate increases, going from top to bottom. Hypothetical coordinates for two sample points are shown to demonstrate these relationships.

DoTempDialog;

\{User enters tape recording temperature and generic file name.

SampleSoundFile;

\{Puts first 5,000 sound bytes into an integer array.

SetTriggerLevel;

\{Plots first 5,000 sound bytes. User clicks mouse on baseline of sound.

ReadSoundFile;

\{Filters and rectifies digitized sound and saves the transformed sound in an integer file. See

Figures 1 and 2.$\}$

SetTriggerLevel2;

\{Plots rectified sound, gets user-selectable trigger level, transforms rectified sound to square pulses. See Figure 1.\}

DistributeIntervals;

\{Measures and accumulates values for pulse duration, pulse period, chirp period.
PlotPulsePeriod, PlotPulseDuration, PlotChirpPeriod; \{Passes several values to the DrawHistograms procedure below.

DrawHistograms;

\{Plots accumulated time intervals in frequency distribution histogram and writes descriptive statistics. See Figure 3.\}

DoSaveDialog;

\{Allows user to save plot as a Macintosh PICT

file as in Figure 3, redo analysis, or exit the SoundAnalysis unit.\}

\section{Detailed Description of the Procedures}

What follows is a detailed description of the relevant procedures in the order in which they are invoked in the main body of the procedure Main (see Appendix). Several procedures are relevant only to the Macintosh operating environment and cannot be implemented in PC environments. Detailed descriptions of these Macintosh-specific procedures are found in procedure headers in the complete source code, which is available from the author.

Procedure Set TriggerLevel. The primary function of this procedure is to get the baseline (ground level = byte value of 123) of the sound. This procedure is not really necessary for digitizers that have AC coupling; but it may be important for the analysis of signals with large DC offsets. The baseline is found by plotting the first 2,500 values of the integer array variable, $\mathrm{Val}$. A static dialogue window (BaseLineDialog resource) is then shown, which asks the user to select the position of the baseline via the mouse. The program is halted until the user clicks the mouse button. The pixel coordinates where the button is clicked are obtained (ROM procedure, getmouse(var X, Trig: integer)) and the integer variable, Trig, is incremented by 5 , which is used for subsequent sound filtering and rectification (see below).

Procedure ReadSoundFile. (See the Appendix for listing of the relevant code.) This procedure reads individual bytes of a previously digitized sound. After reading the sound bytes, it transforms the sound in two steps, which are (1) low-pass filtering of the sinusoidal carrier frequency, and (2) rectification of the sound, which leaves just the positive (above baseline) portion of the amplitude envelope (see Figures 1A, 1B, and 2). This rectified envelope is saved in an integer file (var IntFile2: file of integer) for subsequent interval measurements.

After locking the handle to the soundwave record, sound.wave, the program reads individual bytes ( $\operatorname{var} S B$ : SoundBytePtr) of a soundwave record (var S: SoundWindPtr) that is provided by the SoundWave software. Each byte is the sampled amplitude of the sound (sampling rate of $5.5 \mathrm{kHz}$ ), and since the sound is digitized by an 8-bit digitizer, these amplitude values range between 0 and 255 (a 12- or 16-bit digitizer would also work just as well). For an 8-bit digitizer, an amplitude of 0 corresponds to the maximum upward excursion of the soundpulse amplitude on the monitor screen, a value of 123 corresponds to the baseline (ground) level, and a value of 
G. veletis $7 / 8 / 8720.9 \mathrm{cl}$
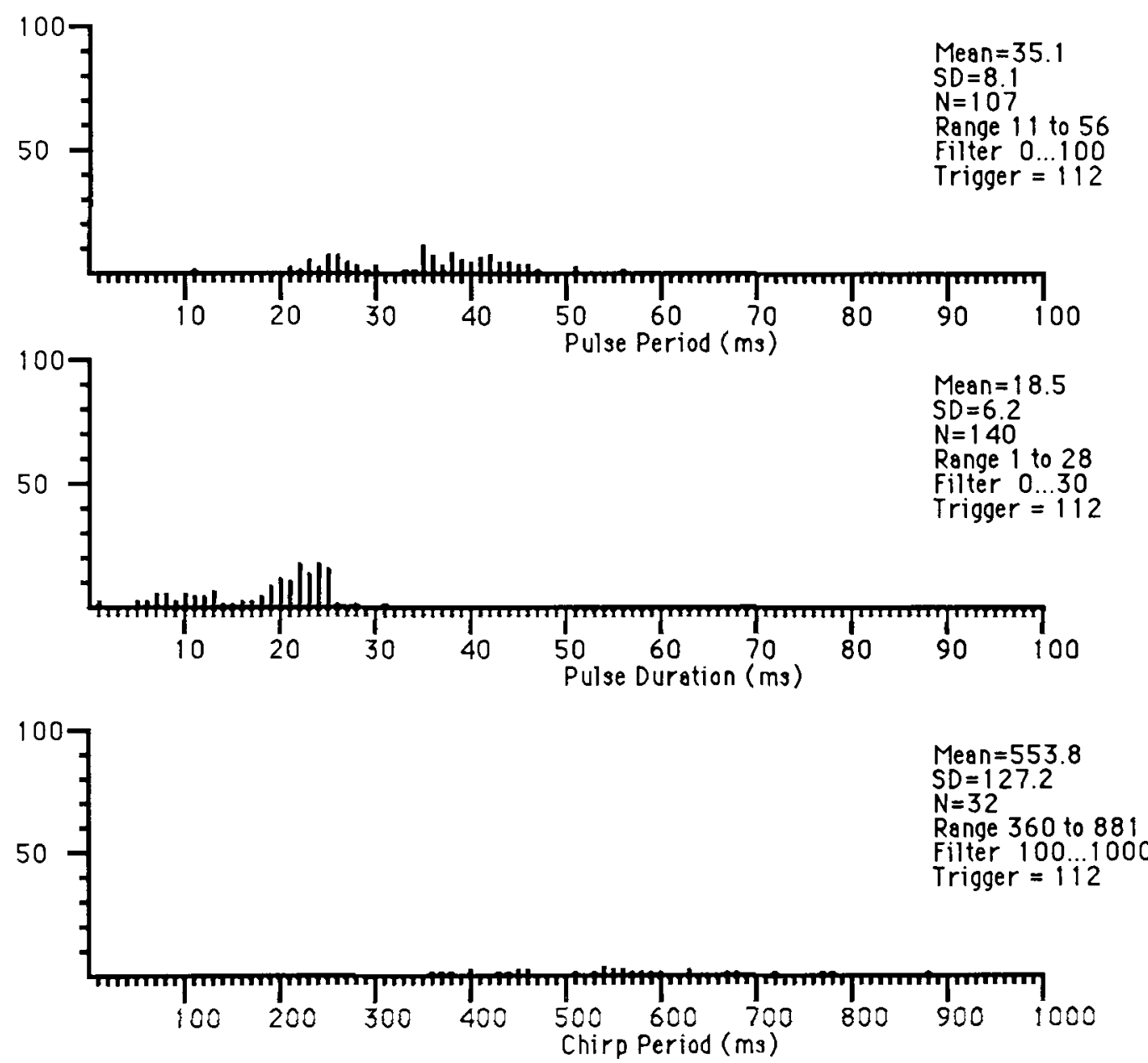

Figure 3. Example of interval histogram plots for pulse duration, pulse period, and chirp period.

255 corresponds to the maximum downward excursion (see Figure 2). Detailed procedures on accessing these values are provided in the SoundWave instruction manual. I emphasize that it is not necessary to read values from a soundwave record; reading amplitude values of any previously digitized sound that has been saved as a simple ASCII text file would work just as well.

The filtering of the sinusoidal carrier frequency is accomplished by downsampling from a $5.5-\mathrm{kHz}$ sampling rate to a rate of $1099 \mathrm{~Hz}$, which is equivalent to a time resolution of $\pm 0.91 \mathrm{msec}$ (reciprocal of rate). This temporal resolution is adequate for the temporal analysis of cricket calling songs. But one should remember that this downsampling approach to low-pass filtering does sacrifice some temporal accuracy. Downsampling is accomplished by writing an amplitude value (stored in the integer variable, Value2) to the integer file, IntFile2, only after five amplitude bytes have been read from the soundwave record.

Sound-pulse rectification is achieved by comparing the current amplitude value (var Value: integer) with the previous amplitude (var Valuel: integer). Remember that the maximum upward excursion of the signal corresponds to an amplitude value of 0 and the maximum downward excursion corresponds to an amplitude value of 255 . So a downward deflection of the sound envelope, as displayed on the monitor screen, is detected if the current amplitude value is greater than the previous value (see Figure 2). The previous amplitude value is stored in the integer variable Value2, and this value is written to the integer file IntFile2, only after five amplitude bytes have been read from the soundwave record (this is the downsampling that has been described above). The variable Value2 is assigned a new value only when another downward excursion of the sound envelope is detected. Negative (below baseline) excursions of the amplitude envelope are eliminated by simply requiring that the current amplitude value, Value, be less than the baseline amplitude Trig, which has been selected previously by the user in the procedure SetTriggerLevel.

Procedure SetTriggerLevel2. (See the Appendix for listing of the relevant code.) This procedure does basi- 
cally four things: (1) It plots the rectified sound pulses on the monitor screen (as in Figure 1B); (2) the user selects a trigger level; (3) the user selects the shortest silent interval between consecutive sound pulses; and (4) the rectified sound pulse envelopes are transformed into simple square pulses (as in Figure 1C) that are stored in another integer file. The trigger level is user-selectable, so that the user can eliminate unwanted background noise and therefore prevent false transformations into square pulses. The selection of the shortest time interval between consecutive pulses is necessary for subsequent calculations of time intervals (i.e., pulse duration, pulse period, chirp period) in the DistributeIntervals procedure (discussed below). The transformation from a rectified soundpulse envelope to a square wave provides definitive pulse rise and fall times for subsequent time-interval measurements in the DistributeIntervals procedure.

The procedure first draws a compressed oscillogram of the transformed sound envelope on the monitor screen. This compressed oscillogram enables the user to accurately adjust the horizontal trigger level so that it is above the level of the background noise. The horizontal trigger level is stored in the integer variable Trig2 and is first drawn across the sound envelope as in Figure 1B. The program then halts and asks the user to adjust the horizontal trigger level, which can be moved up or down via buttons in the dialog window (TrigDialog resource) to eliminate background noise that may confound precise transformations of rectified sound-pulse envelopes to square pulses. The horizontal trigger level is incremented or decremented in 2-pixel increments (var TrigIncrement: integer). Once the horizontal trigger is set correctly by the user, two rows of noncompressed, rectified pulse envelopes are redrawn on the monitor along with the newly set horizontal trigger level. The program halts, and the user is then asked in a new dialog window (PulseDia$\log$ resource) to select the shortest silent interval between consecutive pulses by clicking the mouse at the end of one pulse and at the beginning of the next consecutive pulse. The procedure getmouse returns the pixel coordinates where the mouse button was clicked (the coordinates are (PDBegin, dummy) and (PDEnd, dummy)). The integer variable BaseLineCriterion stores either one half of the absolute value of the silent interval between PDBegin and PDEnd or a minimum value of 5 . The value stored in BaseLineCriterion is used in searches for true baseline conditions (i.e., silent intervals) between consecutive pulses, which are necessary for the calculation of consecutive time intervals in the DistributeIntervals procedure. The pulse envelope is then transformed into simple square pulses, with the vertical rise and fall times occurring at the point where the sound envelope intersects the horizontal trigger level (as in Figure 1C). The square pulses are stored in another integer file (var IntFile3: file of integer).

Procedure DistributeIntervals. (See the Appendix for listing of the relevant code.) This procedure identifies the beginnings and ends of square pulses. These rise and fall times of the square pulses are then used to calculate pulse durations, pulse periods, and chirp periods (see Figure 1A for definitions of these temporal pattern intervals). Values for these intervals are then stored in their respective arrays (var IntervalPP, IntervalPD, IntervalCP: array $[0 . .700]$ of integer) for subsequent analysis by the DrawHistograms procedure. Temporal parameter intervals are calculated only when a true baseline condition (i.e., silent interval) between consecutive square pulses is detected by the program. The criterion for a true baseline condition is user-selectable in the SetTriggerLevel2 procedure. The minimum criterion duration for a true baseline condition is stored in the integer variable BaseLineCriterion.

The square pulses along with graphical indications of pulse rise and fall conditions (shown by small solid and open circles for pulse rise and fall time, respectively; large solid circles for chirp rise time) are displayed on the monitor (see Figure 1C). After the entire screen of the monitor is filled with several rows of square pulse oscillograms, a dialogue box (SkipDialog resource) allows the user to (1) continue both the analysis and the oscillogram plots, (2) continue just the analysis without the oscillogram (the boolean variable Skip is made TRUE), or (3) exit the analysis and reselect the trigger level (the boolean variable Redo is made TRUE; see Main of the Main procedure in the Appendix).

The following description of the DistributeIntervals procedure starts at the Main section of the procedure (the relevant code is found in the Appendix). Several integer and file variables are first initialized, and then the drawing window (var DrawWindow: WindowPtr) is shown. The first value in the integer file IntFile 3 is stored in the integer variable CurValue. All subsequent integers in the IntFile 3 file are read in the main repeat loop until an endof-file condition exists $(E O F($ IntFile3) $=$ TRUE). This repeat loop is also exited either when the integer array sizes have been exceeded (NPP, NPD, or NCP equal to ArrayMax) or when the boolean variable Redo has been set to TRUE in the DrawPulses subprocedure. There are several IF statements in the main repeat loop that compare consecutive square pulse amplitudes with the trigger level established in the SetTriggerLevel2 procedure. At the end of the main repeat loop, the DrawPulses subprocedure is invoked for the graphical display of the square pulses. The action of each IF statement in the repeat loop is now described separately.

IF Statement \#1. The routines under this conditional IF statement test for a true baseline condition (silent interval) between consecutive pulses. The current and previous amplitudes of square pulses are compared with the trigger level, which is stored in the integer variable Trig. If both of these amplitudes are greater than the trigger level (i.e., a baseline condition), then more amplitudes are read until either a rise above the trigger level is detected or a baseline condition has existed for a minimum 
length of time. The minimum time is stored in the integer variable BaseLineCriterion, in the procedure SetTriggerLevel2. Remember that these routines test for a criterion baseline duration only after a rise above trigger level is encountered. A search for baseline will not occur when a baseline condition already exists (i.e., when Baseline: =TRUE). The boolean variable Baseline is set to FALSE only when a pulse rise is detected in IF Statement $\# 2$

IF Statement \#2. In this conditional statement, the time of a pulse rise time will be stored only when two conditions are TRUE-when (1) a rise above trigger level has been detected (CurValue < Trig and PrevValue > Trig), and (2) a baseline condition has just been encountered (Baseline: =TRUE). The boolean variable Baseline prevents the detection of a false rise time. For example, in Figure 1C, the time of the second rise in Pulse 1 is not stored, because the duration of the short baseline (silent interval) that precedes this second rise is not greater than a minimum criterion duration, which is stored in the integer variable BaseLineCriterion. The current value of the sample interval counter, $y$, is stored in the integer variable Rise. Boolean values are assigned to Up1 and Up2 for subsequent calculations of pulse and chirp periods in IF Statement \#5. If the pulse rise time is the first one encountered, then Up1 is made TRUE and the value of the sample interval counter, $y$, is stored in the integer variable FirstRise. If the rise time is the beginning of the next consecutive pulse, then Up2 is made TRUE. The procedure ends by assigning FALSE values to the two boolean variables Baseline and PDCalculated.

IF Statement \#3. The routines under this conditiona' statement find the end of the pulse. The time of a pulse fall is stored only when a fall below trigger level has been detected (CurValue $>$ Trig and PrevValue $<$ Trig) and a baseline condition has not been detected (Baseline:=FALSE). The value of the sample interval counter, $y$, is stored in the integer variable Fall.

IF Statement \#4. Pulse duration (=Fall - Rise) will be calculated only when a baseline condition exists (Baseline:=TRUE) and the boolean PDCalculated is FALSE. The PDCalculated boolean prevents the calculation of pulse duration before the next pulse rise condition is encountered, after which the booleans Baseline and PDCalculated are both set to FALSE in IF Statement \#2. The pulse duration is calculated in terms of differences between sample interval values, which will subsequently be converted to milliseconds in the DrawHistograms procedure. Pulse duration is stored in the integer array IntervalPD.

IF Statement \#5. Pulse period (or chirp period) is calculated by the routines under this conditional statement only when two consecutive pulse rise times have been encountered (i.e., when the booleans Upl and Up2 are both TRUE). Pulse period is stored in the integer array IntervalPP, crily if the value of the expression Rise-FirstRise is less than a predetermined, minimum value for chirp period (var MinCP: integer). If Rise-FirstRise is greater than this minimum value, then a chirp period is calculated by subtracting the chirp rise time (var ChirpRise: integer) from the current rise time (var Rise: integer). The chirp period is stored in the integer array, IntervalCP, only if the chirp period is less than a predetermined, maximum value for chirp period (var MaxCP: integer). After a chirp period is stored, several variables are reinitialized to prepare for the detection of the next pulse rise. These variable reinitializations are Chirprise: $=$ Rise (the current rise time), FirstRise: $=$ Rise, and Up2: $=$ FALSE.

\section{Concluding Comments}

The sound analysis program described above can rapidly measure temporal pattern intervals of repetitive sounds that have been previously digitized. The digitized sound is transformed by filtering out the carrier frequency and rectifying the sound pulses, which results in a sound file that shows just the top half of the sound-pulse envelope. The program is flexible, because it allows the user to visually adjust trigger levels and to select the minimum silent interval between consecutive sound pulses, which is necessary for automatic measurements of sound-pulse intervals and the avoidance of false pulses inherent in many recordings of animal sounds. The program is userfriendly, due to the implementation of dialogue boxes and oscillograms, which are under mouse control. The program is written in THINK Pascal and is currently imbedded in a commercially available, sound digitizing and analysis program for the Macintosh computer (SoundWave, Impulse, Inc.). However, the use of the sound temporal pattem analysis program is certainly not restricted to this hardware/software environment. The algorithms described in this paper can be incorporated in any hardware/software environment for sound digitization and analysis. The program can be modified to input any digitized sound file that has been saved previously as an ASCII file.

The temporal pattern analysis program can be used on any repetitive, pulsatile sounds that have amplitude-time envelopes like those shown in Figure 1A. The oscillogram in Figure $1 \mathrm{~A}$ shows a cricket calling song with a $5-\mathrm{kHz}$ carrier frequency, but this oscillogram could just as easily represent repetitive sounds produced by other acoustic insects such as grasshoppers, katydids, or cicadas, as well as by anurans, birds, and mammals. Low-pass filtering of the $5-\mathrm{kHz}$ carrier frequency in cricket sounds is achieved in the temporal analysis program by downsampling from $5.5 \mathrm{kHz}$ to $1099 \mathrm{~Hz}$ by skipping five amplitude bytes of the soundwave record (see desctiption of ReadSoundFile procedure). Fewer or more bytes could be skipped in this procedure to achieve the same downsampling effect for repeated, pulsatile animal sounds that have lower or higher carrier frequencies. More information about downsampling as a form of filtering can be found in Oppenheim and Schafer (1975) and Sterns and David (1988). 
The method of signal rectification described in the temporal analysis program is nonstandard, which may result in temporal measurement accuracies (i.e., pulse duration) that are less than those obtained using more standard methods. A rectified signal is normally computed by taking the absolute value of the signal, which assumes that the signal amplitude varies around 0 (see $O$ 'Shaughnessy, 1987). The rectification that I have described eliminates the lower half of the signal (amplitudes greater than 123) and assumes that the signal amplitude varies between 0 and 255. This means that finer details of the amplitude envelope may be lost, but accurate measures of pulse onset and offset times are unaffected. Reduced measurement accuracies may become slightly more prevalent for signals that have carrier frequencies of less than $1 \mathrm{kHz}$, which corresponds to signal periods greater than $1 \mathrm{msec}$.

The selectable trigger level allows the user to eliminate background noise. Sounds that have low signal-to-noise ratios with significant background noise (i.e., wind noise or noise produced by other acoustic animals) can be analyzed by the program if one moves the horizontal trigger level above the peak amplitude of background noise (see the SetTriggerLevel2 procedure). This has been done on cricket song recordings that have high background noise levels. Measurements of temporal pattern periodicities such as pulse and chirp period remained accurate, but measurements of pulse durations were consistently short and inaccurate, because the exact onset and offset times of individual sound pulses were hidden by the background noise.

The measurement algorithms described in this paper could be modified to analyze repetitive sounds that are not pulsatile. For example, the algorithms could be modified to analyze constant, nonpulsatile spectral properties such as fundamental, harmonic, and dominant frequencies. This could be done by including additional trigger- level selection routines that allow the user to select the peak amplitudes that are correlated with these spectral properties. One could, for example, select the peak amplitude in the expanded sound oscillogram that is correlated with fundamental frequency and use this amplitude as a trigger level for measuring the instantaneous or average periodicities of the fundamental. Higher harmonics could be measured from the wave form in a similar manner. This measurement strategy would work only on acoustic signals that have unchanging relative amplitudes-an attribute that is common in continuous sound signals produced by some acoustic insects and anurans. Spectral analysis of more complex sounds that have changing amplitudes and frequencies, such as the sounds produced by some birds and mammals, would most likely require additional fast Fourier transform routines.

\section{Program Availability}

The complete source code is available from the author. Those wishing to receive copies of the code via electronic mail may contact the author at DOHERTY @VUVAXCOM.

\section{REFERENCES}

Doherty, J. A., \& Hoy, R. R. (1985). Communication in insects. III. The auditory behavior of crickets; Some views of genetic coupling. song recognition, and predator detection. Quarterly Review of Biology, 60, 457-472.

Huber, F., Thorson, J. (1985). Cricket auditory communication. Scientific American, 253, 60-68.

OPPENHEIM, A. V., SCHAFER, R. W. (1975). Digital signal processing. Englewood Cliffs, NJ: Prentice-Hall.

O'Shaughnessy, D. (1987). Speech communication. Reading, MA: Addison-Wesley.

STERNS, S. D., \& DAVID, R. A. (1988). Signal processing algorithms. Englewood Cliffs, NJ: Prentice-Hall. 


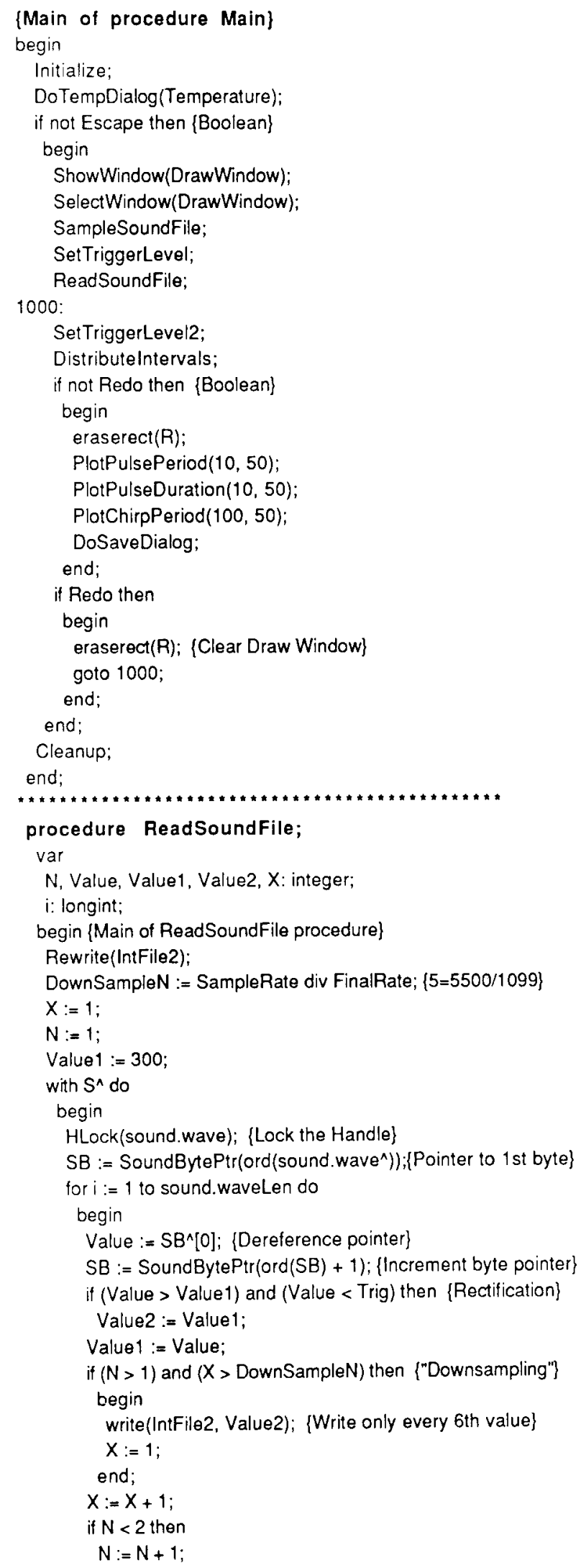

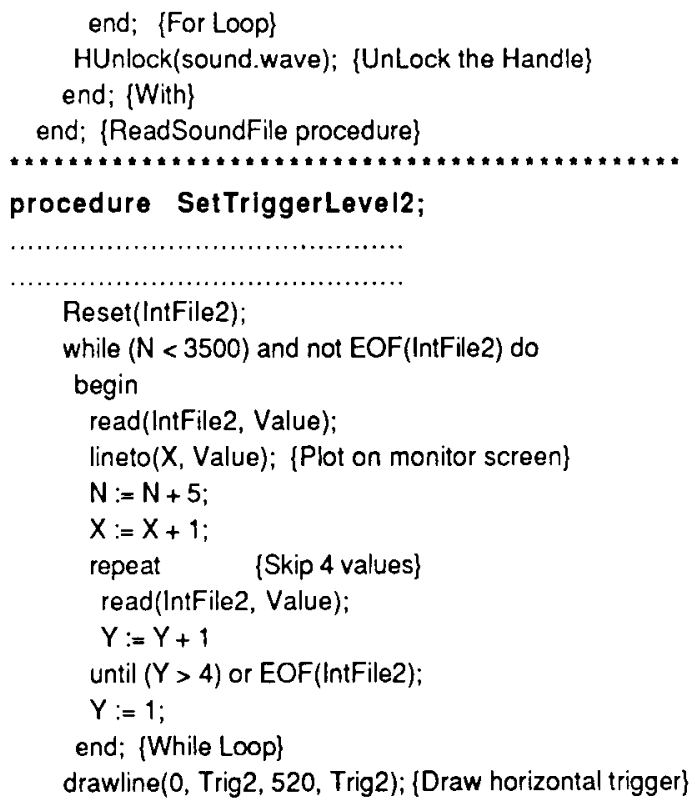

\{Find shortest silent period between consecutive pulses\} ShowWindow(windowptr(PulseDialog));

SelectWindow(windowptr(PulseDialog)); DrawDialog(PulseDialog);

repeat $\{$ Wait until mouse button is pressed until button;

getmouse(PDEnd, dummy); paintcircle(PDEnd, dummy, 2);

\{Pause program execution for 1 second Delay(60, Time);

repeat \{Wait until mouse button is pressed\} until button; getmouse(PDBegin, dummy); paintcircle(PDBegin, dummy, 2); Delay(60, Time);

\{Set value of integer variable, BaseL ineCriterion\} if (abs(PDBegin - PDEnd)) $<5$ then

BaselineCriterion $:=5$

else

BaseLineCriterion := (abs(PDBegin - PDEnd) div 2); HideWindow(windowptr(PulseDialog));

\{Tranpose digitized waveform into simple square pulses\} Trig := Trig2; \{Save for "Distributelntervals" procedure\} $Z$ := Trig2 - 50; \{Top of the square pulse\} $T:=$ Trig2 + 5; $\{$ Bottom of the square pulse Reset(Intfile2);

Rewrite(Intfile3);

while not eof(IntFile2) do begin read(Intfile2, Value); if Value >x Trig2 then write(Intfile3, T) else write(Intfile3, Z); end; \{While Loop\} end; \{SetTriggerLevel2 procedure\} 
APPENDIX (Continued)

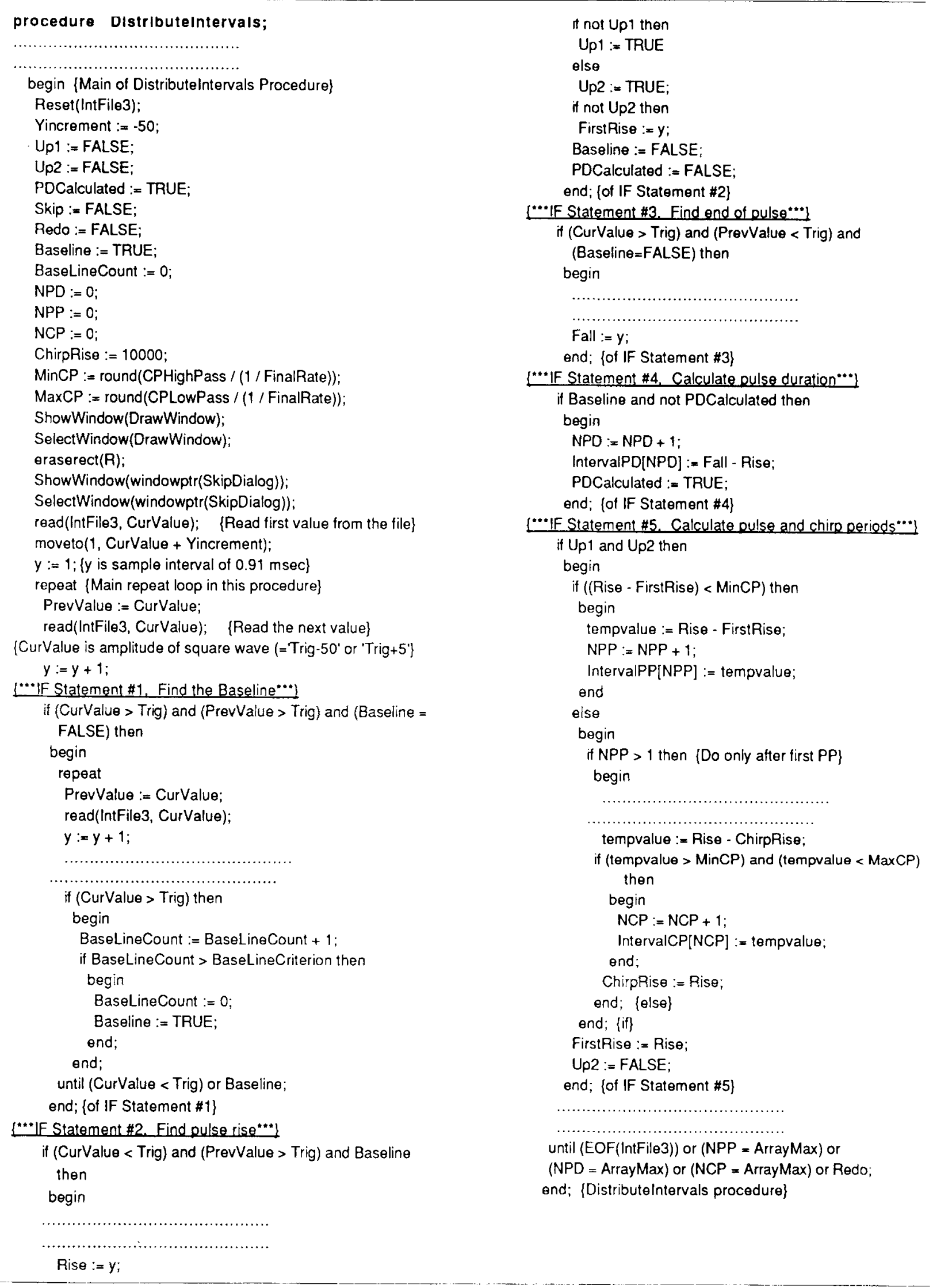

